\title{
Correction: Division time-based amplifiers for stochastic gene expression
}

Cite this: Mol. BioSyst., 2016, 12,678

DOI: $10.1039 / \mathrm{c} 6 \mathrm{mb} 90004 \mathrm{f}$

www.rsc.org/molecularbiosystems

\author{
Haohua Wang, ${ }^{\text {ab }}$ Zhanjiang Yuan, ${ }^{a}$ Peijiang Liu ${ }^{a}$ and Tianshou Zhou*a
}

Correction for 'Division time-based amplifiers for stochastic gene expression' by Haohua Wang et al., Mol. BioSyst., 2015, 11, 2417-2428.

The authors regret using data from ref. 42 (E. D. Hawkins, J. F. Markham, L. P. McGuinness and P. D. Hodgkin, Proc. Natl. Acad. Sci. U. S. A., 2009, 106, 13457-13462) without the permission of the authors and publisher. Here, we clearly indicate in the legend of Fig. 9 that the data presented in this figure are reproductions of Fig. 1(F) of ref. 42 and are used with the permission of the authors and publisher.
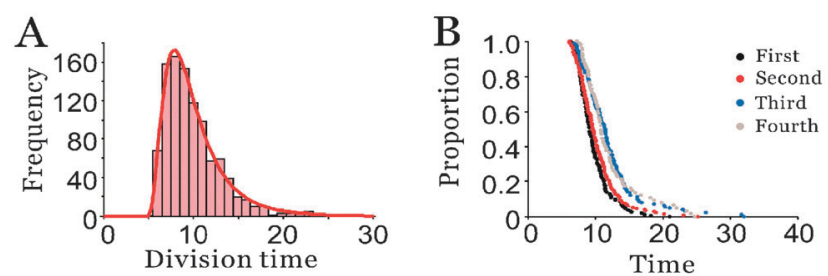

Fig. 9 Experimental data for variation in subsequent B-cell division times represented as a histogram collated in $1 \mathrm{~h}$ time intervals: (A) the distribution of division times, where the solid line represents a fitted lognormal distribution with the mean $\tau=9.3$ and the variance $\sigma=2.54$; (B) the proportion of division events, where "First" represents the first division (similarly for the others). The data presented in this figure are reproductions of Fig. 1(F) of ref. 42 and are used with the permission of the authors and publisher.

The Royal Society of Chemistry apologises for these errors and any consequent inconvenience to authors and readers.

\footnotetext{
${ }^{a}$ School of Mathematics and Computational Science, Sun Yat-Sen University, Guangzhou 510275, People's Republic of China. E-mail: mcszhtsh@mail.sysu.edu.cn

${ }^{b}$ Department of Mathematics College of Information Science and Technology Hainan University, Haikou 570228, People's Republic of China
} 